\title{
Pathophysiology of streptokinase-induced hypotension in acute myocardial infarction: a systematic review of clinical evidence
}

Karniza Khalid, ${ }^{1,2}$, Raja Elina Ahmad ${ }^{1}$, Alwin Y.H. Tong ${ }^{3}$, Sze Yee Lui ${ }^{4}$, Ida Zaliza Zainol Abidin ${ }^{4}$

${ }^{1}$ Department of Physiology, Faculty of Medicine, University of Malaya, Kuala Lumpur, Malaysia

${ }^{2}$ Clinical Research Centre, Hospital Tuanku Fauziah, Perlis, Ministry of Health Malaysia, Malaysia

${ }^{3}$ Department of Internal Medicine, Hospital Tuanku Fauziah, Perlis, Ministry of Health Malaysia, Malaysia

${ }^{4}$ Emergency and Trauma Department, Hospital Tuanku Fauziah, Perlis, Ministry of Health Malaysia, Malaysia

Submitted: 29 December 2020

Accepted: 6 March 2021

Arch Med Sci Atheroscler Dis 2021; 6: e85-e94

DOI: https://doi.org/10.5114/amsad.2021.105410

Copyright @ 2021 Termedia \& Banach

\section{Abstract}

Introduction: Despite the common occurrence of streptokinase-induced hypotension among patients with acute myocardial infarction, the underlying pathophysiology remains obscure and poorly understood. Our study aimed to pool clinical evidence on the potential mechanism of streptokinase-induced hypotension through a systematic review of the literature.

Material and methods: We conducted literature search from Medline, Scopus and Web of Science on clinical studies related to streptokinase-induced hypotension.

Results: Our search yielded 972 citations. After removal of duplicates, 878 articles were screened for eligibility, of which 856 papers were excluded due to various reasons. Of the remaining 22 articles retrieved with full texts, eight relevant articles were selected for final analysis. Three themes emerged as the proposed mechanisms of streptokinase-induced hypotension, including (i) reduction in total peripheral resistance, (ii) complement activation, and (iii) dismissal of hypotheses involving other intermediaries.

Conclusions: Our findings suggest that the underlying mechanism of streptokinase-induced hypotension lies primarily in the reduction in total peripheral resistance.

Key words: streptokinase, blood viscosity, vascular resistance, complement activation, myocardial infarction.

\section{Introduction}

Streptokinase-induced hypotension is a common adverse drug reaction related to streptokinase therapy given to patients with acute myocardial infarction [1, 2]. Despite the continuous research that dates back to the early 1970s, the mechanism of streptokinase-induced hypotension is still poorly understood and is believed to be a rate-related phenomenon [3].

A plethora of research conducted in laboratory and animal studies do provide the basis for understanding, however the results of which were

\author{
Corresponding author: \\ Karniza Khalid MBBS \\ Department of Physiology \\ Faculty of Medicine \\ University of Malaya \\ 50603 Kuala Lumpur \\ Malaysia \\ E-mail: karniza.khalid@moh. \\ gov.my
}


not immediately transferrable to humans. Studies with specific interest in the clinical outcomes of streptokinase therapy in patients with acute myocardial infarction were conducted with variable study designs that span from observational cohort study [4-6] to double-blind randomised controlled trial [7-9]. However, the studies were conducted with different treatment regimens of streptokinase therapy and needed to be interpreted with caution. To the best of our knowledge, there is no cumulative clinical evidence to suggest potential mechanisms leading to streptokinase-induced hypotension in patients with acute myocardial infarction. Understanding the concept and its underlying pathophysiology will provide clinicians with a thorough grasp on how the therapy affects susceptible patients and the suitable practice to avoid this complication.

Hence, our study aimed to compile, appraise and synthesize the plausible mechanism of streptokinase-induced hypotension in acute myocardial infarction through a systematic review of the literature.

\section{Material and methods}

Comprehensive online literature search was conducted through three main medical databases (Medline, Scopus and Web of Science) for published articles and book chapters on specified key terms. Forward-and-backward citation tracing was also conducted to ensure maximal retrieval of possible relevant references.

\section{Search key terms}

(Streptokinase OR streptase) AND (hypotension OR "low blood pressure") with a restriction applied to the title/abstract. The search was completed on 14 October 2019.

\section{Inclusion and exclusion criteria}

The study only included clinical studies documented in articles, reviews and book chapters on streptokinase-induced hypotension among patients with acute myocardial infarction; reported in the English language; of any study designs; with or without intervention, inclusive of primary, secondary or tertiary care or non-governmental provisions. We exclude articles without primary emphasis to delineate the underlying pathophysiology of streptokinase-induced hypotension. This included studies that discussed the clinical management of acute myocardial infarction, the frequency of side effects of administered streptokinase therapy among patients with acute myocardial infarction and studies on clinical prognosis of patients with acute myocardial infarction receiving streptokinase.

\section{Article selection, review and extraction}

Eligible documents were retrieved and exported to Mendeley Desktop Ver. 1.19.4. Removal of duplicates was done through the software and further review of the titles and abstracts were independently done by two authors (KK \& AT). Disagreements with regards to article eligibility were reconciled through discussion.

Prior to analysis, the finalised literature was critically appraised by one reviewer (KK), who has a primary interest in cardiac physiology. Retained references were divided into themes (i.e. main pathway) and the full texts were critically analysed and appraised (KK). Information collected includes: (i) study identifier (author and year of publication); (ii) study design (setting and dataset); (iii) participants; (iv) results, and (v) conclusions.

\section{Data analysis}

The result was tabulated according to the thematic content (i.e. emerging new findings or concepts, approval or disapproval of previous hypotheses). A concise flowchart diagram incorporating all the proposed mechanisms in the literature was constructed as a visual aid.

\section{Ethical clearance}

The research was registered with the National Medical Research Registry of the Ministry of Health, Malaysia (NMRR-19-2871-50953) and received ethical clearance from the Medical Research and Ethics Committee (MREC), Ministry of Health, Malaysia (KKM/NIHSEC/P19-2421(5)).

\section{Results}

The search retrieved 972 citations based on the specified key terms with a restriction to the title/abstract. After removal of duplicates, 878 articles were screened for eligibility. Based on the pre-established criteria that only human studies published in the English language were considered for this review, a total of 856 articles were further excluded (839 were considered irrelevant to the study objective, 10 were animal or in vitro studies, and seven were reported in the language other than English). Of the remaining 22 articles that have been retrieved with the full texts, 14 were excluded due to their lack of relevance to the discussion of the mechanism(s) underpinning streptokinase-induced hypotension. PRISMA flowchart of the article selection for critical appraisal is depicted in Figure 1.

Majority of the papers that were excluded from the final review mainly report on the clinical aspects of streptokinase administration in patients with acute myocardial infarction, such as on the 


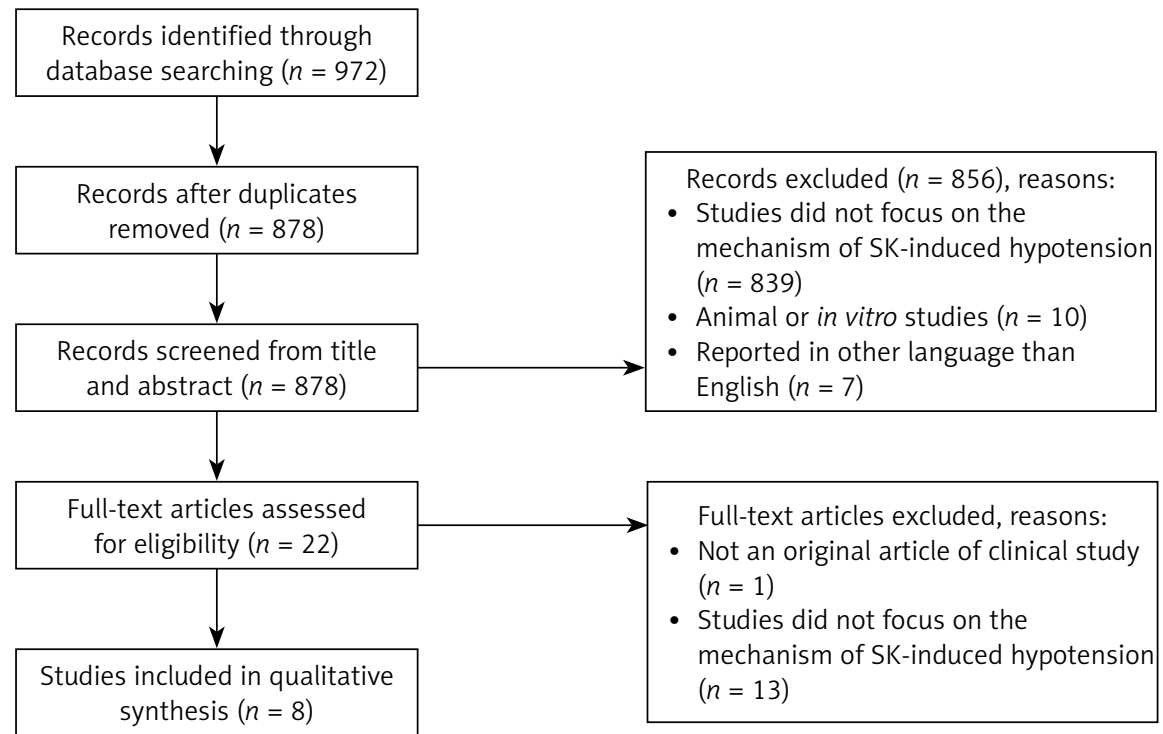

Figure 1. PRISMA flowchart of the study selection

duration of the hypotensive episode, the average drop in blood pressure and remedial clinical actions taken but did not discuss the underlying possible mechanism of its development. A total of eight clinical studies were eventually included in the analysis. A summary of the relevant studies is tabulated below (Table I).

There were three themes that emerged as the proposed mechanism to explain streptokinase-induced hypotension in patients with acute myocardial infarction receiving intravenous streptokinase therapy. This includes (i) reduction in total peripheral resistance as the main pathophysiology underpinning streptokinase-induced hypotension, (ii) complement activation resulting in widespread arteriolar dilatation, and (iii) the dismissal of hypotheses involving other intermediaries.

\section{Reduced total peripheral resistance as the main pathophysiology underpinning streptokinase-induced hypotension}

A controlled clinical trial by Neuhof et al. found a significant decrease in total peripheral resistance averaging up to $21 \%$ following treatment with intravenous streptokinase [10]. In their study, the total peripheral resistance was measured through invasive real-time monitoring and was computed from the oxygen uptake, arteriovenous oxygen difference and the difference between arterial and central venous blood pressure obtained from centrally-placed transducers. Additionally, they also found that there was a concurrent increase in the cardiac output during the hypotensive episode which has been attributed to the autonomic response that is highly dependent on the myocardium functional capacity during the attack.
However, the paper did not specify the calculation used to obtain the total peripheral resistance in their study.

\section{Complement activation during streptokinase therapy results in widespread arteriolar dilatation}

Several clinical studies have found a significant increase in the complement levels within 30 min of intravenous streptokinase infusion in patients with acute myocardial infarction [4, 11]. For example, Frangi et al. found a significant increment in all anaphylatoxin levels up to 10 times the baseline values in patients given streptokinase, as compared to patients who did not receive the treatment. Similarly, Pachaii et al. also found a significant increase in C3d level to more than $200 \%$ of the baseline values within 30 min of intravenous streptokinase infusion in patients with hypotension. This phenomenon also heralds transient leukopenia suggested to be due to neutrophil sequestration commonly seen with complement activation [4].

However, despite the synonymous evidence of complement activation in patients given streptokinase, Frangi et al. and Pachaii et al. did not find any linear relationship between the degree of complement activation and the degree of the induced hypotension episode. Furthermore, Skopál et al. asserted that the activation of the complement system by streptokinase is related to the contaminants present in the various formulation of different streptokinase products rather than the direct effect of streptokinase itself.

This suggests that the hypotensive episode commonly seen with streptokinase therapy may only be partially explained through complement 


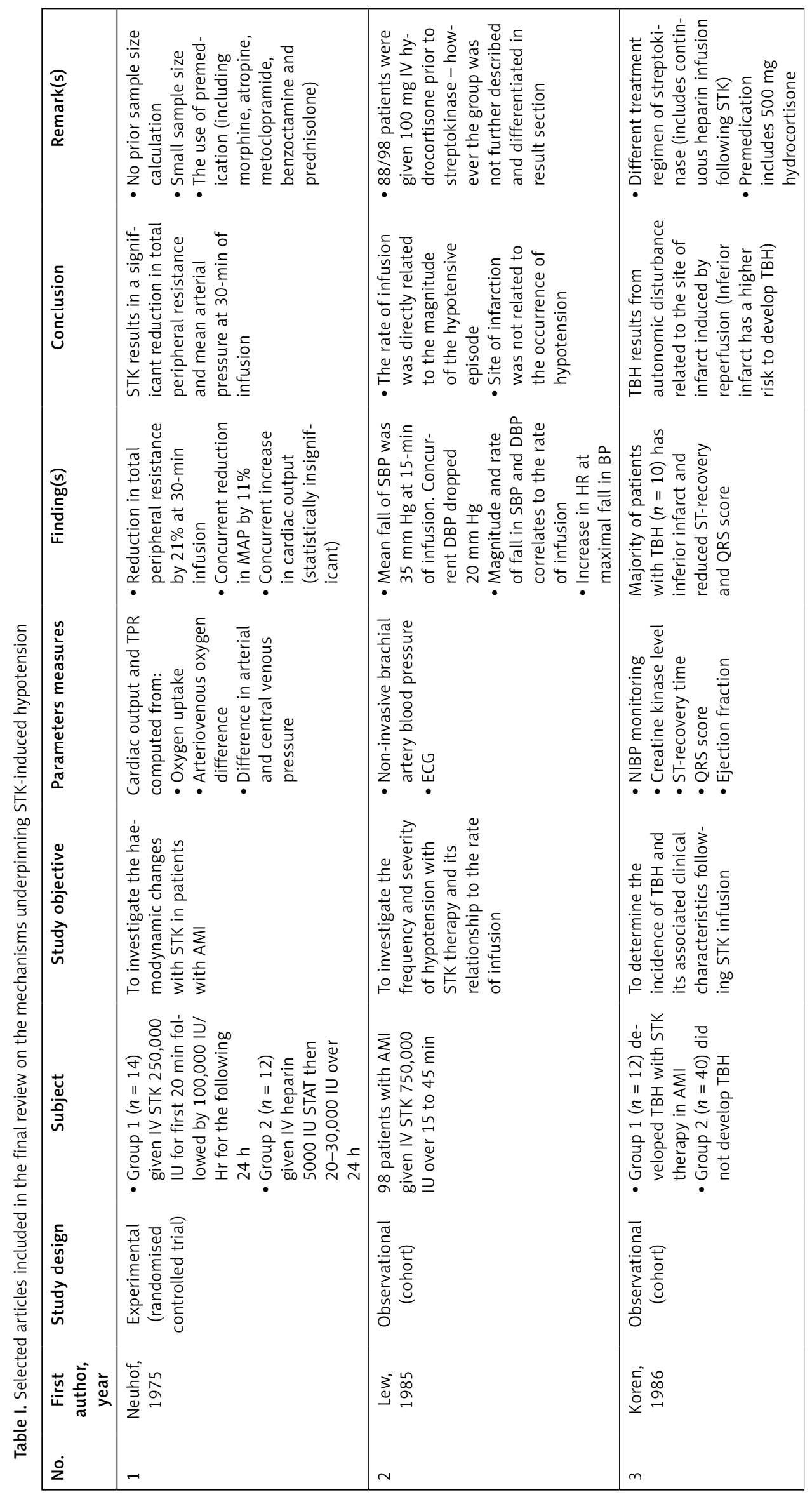




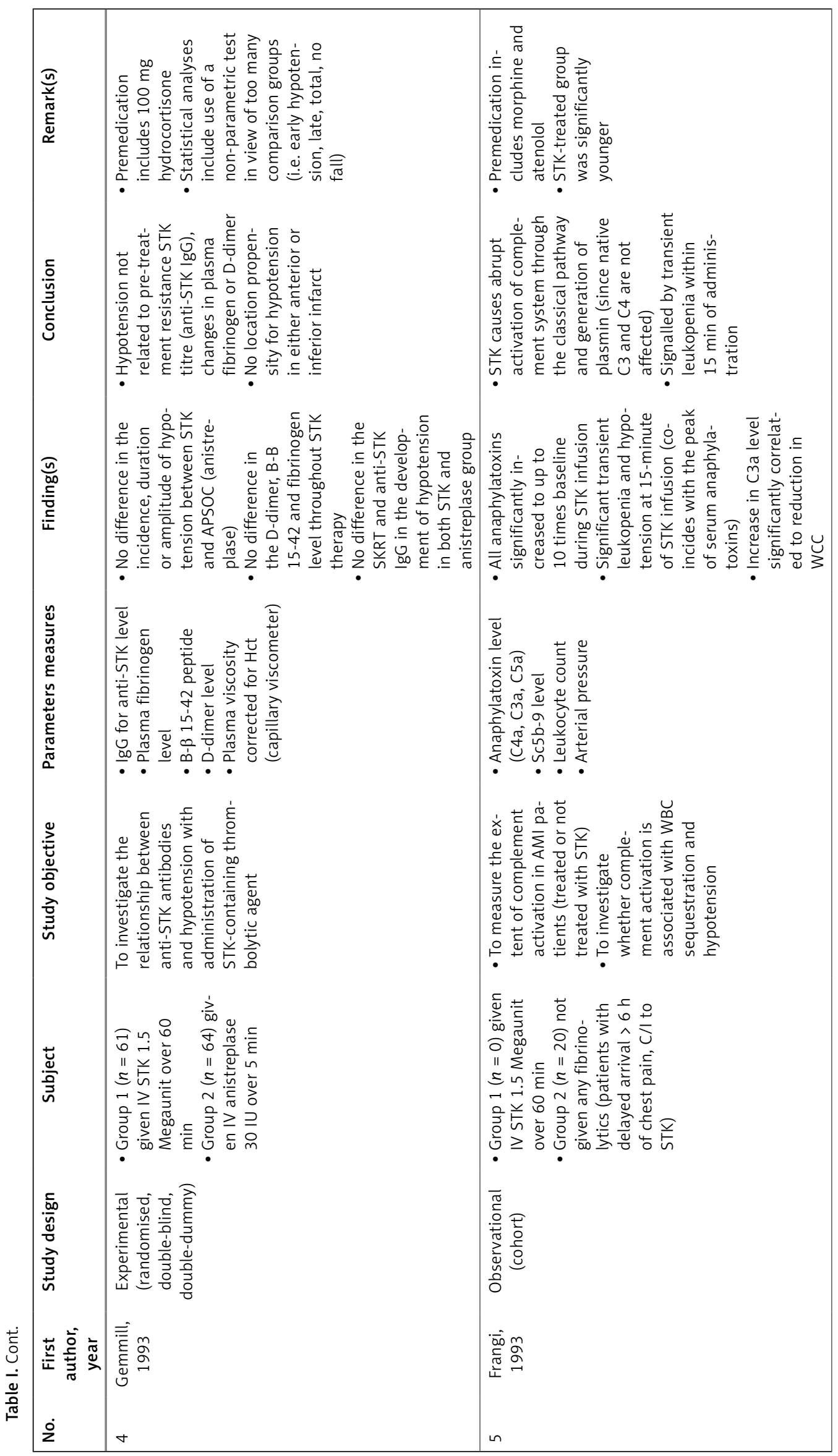




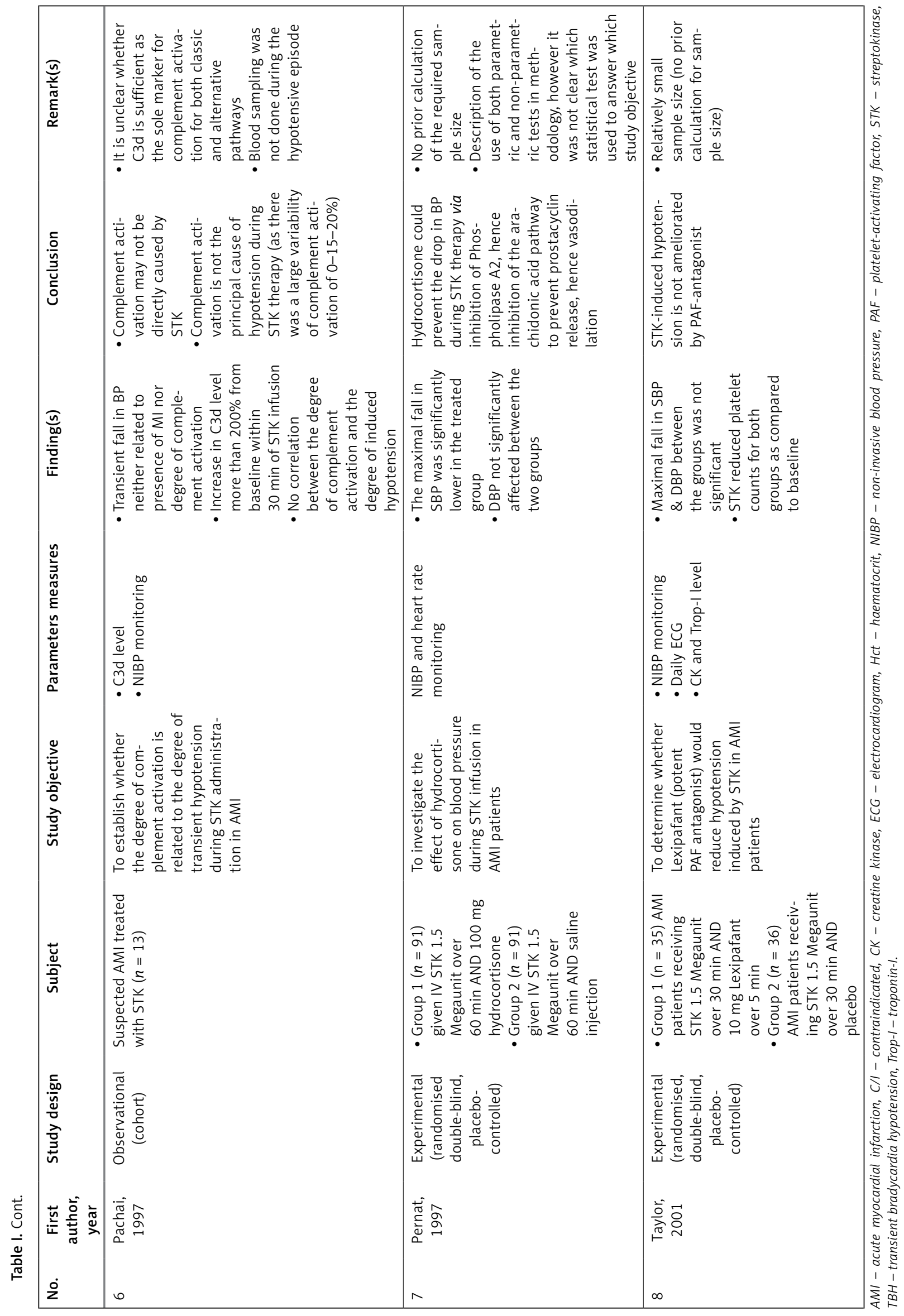


activation, and not the principal cause of streptokinase-induced hypotension.

\section{Dismissal of other hypotheses involving other intermediaries of streptokinase- induced hypotension}

Other hypotheses postulated for the mechanism of streptokinase-induced hypotension include the (i) direct action of streptokinase, (ii) the action of plasmin, (iii) the action of fibrin-degradation product, (iv) the action of factor XII that is activated by plasmin, and (v) as part of an immunological reaction to streptokinase antigenic property.

Streptokinase has been proposed to directly stimulate intravascular synthesis of platelet-activating factor (PAF) in patients with acute myocardial infarction, that is known to cause vasodilation. However, administration of Lexipafant, a potent PAF-receptor antagonist did not sufficiently prevent the occurrence of hypotension in patients with acute myocardial infarction receiving streptokinase [9].

Apart from that, the reduction in total peripheral resistance was also presumed to be contributed by streptokinase reaction cascade resulting in non-fibrin specific lysis of systemic fibrinogen, an important variable of blood viscosity. However, this is not the case as Gemmill et al. found no relationship between the B- $\beta$ 15-42 peptide, a degradation fragment of fibrinogen and $D$-dimer, with the corrected plasma viscosity and the incidence of hypotension during streptokinase therapy.

On the other hand, plasmin produced from the cascade of Streptokinase reaction may activate the kallikrein system to release bradykinin, a potent endothelium-dependent vasodilator. Plasmin is also known to activate factor XII that is known to convert the first component of the complement system through the alternative pathway and has also been implicated in the conversion of high molecular weight kininogen into bradykinin. However, these suggested mechanisms have yet to be proven in vivo and remain hypothetical.

Fibrin-degradation product (FDP) has been studied in vitro to activate the arachidonic acid pathway with the consequent release of prostacyclin, a potent vasodilator. Hence, the administration of the glucocorticoid may, in theory, prevent the hypotensive episode by inhibiting the phospholipase A2 enzyme, thus inhibiting the arachidonic acid pathway. However, Pernat et al. did not find a conclusive result as the magnitude of the difference in systolic blood pressure between the treatment and placebo groups was too small to be clinically significant when intravenous hydrocortisone $100 \mathrm{mg}$ was given in a double-blind placebo-controlled study involving patients with acute myocardial infarction given Streptokinase.

Streptokinase-induced hypotension was also presumed to be immunologically mediated possibly due to IgE class antibodies in patients with prior exposure to streptokinase. However, Gemmill et al. did not find any relationship between the baseline streptokinase resistance titre and the baseline anti-streptokinase IgG concentration with the occurrence of hypotension during streptokinase therapy.

Figure 2 presents the summary of cumulative evidence from clinical studies on the pathophys-

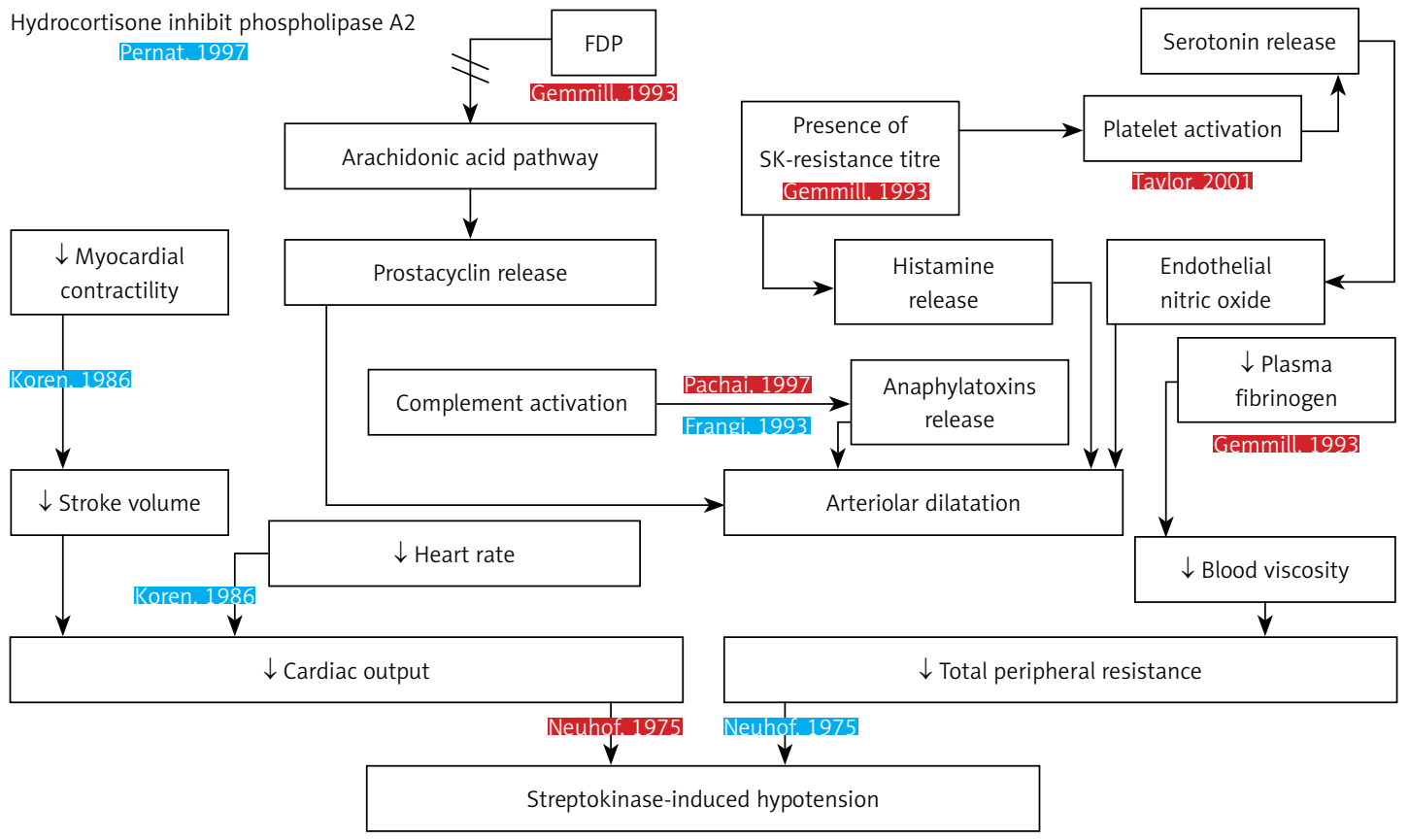

Figure 2. Summary of clinical trials underpinning streptokinase-induced hypotension 
iology of streptokinase-induced hypotension in patients with acute myocardial infarction.

\section{Discussion}

Our review found only eight relevant articles investigating the potential mechanisms underlying streptokinase-induced hypotension, despite hypotension being a well-recognised side effect of streptokinase therapy that has been still poorly understood to date $[12,13]$. A vast majority of the scientific investigation on the pharmacokinetics of streptokinase was based on in vitro and animal studies, which findings may not be directly transferrable to humans.

Streptokinase-induced hypotension is a wellknown side effect of intravenous streptokinase therapy in acute myocardial infarction [12]. Despite its mild and transient course that is typically reversible with Trendelenburg position or fluid challenge, it can produce an undesirable effect by interfering with streptokinase fibrinolytic activity as the decrease in perfusion pressure during the hypotensive episode restricts the circulation of streptokinase in reaching the targeted sites. Furthermore, the state of the ischaemic myocardial tissue is further compromised by the reduction in the collateral flow to the ischaemic myocardial tissue during the hypotensive episode [14, 15].

Knowledge on the mechanism underlying streptokinase-induced hypotension will potentially allow pharmacological modulation and promote vigilance in clinical practice. We conclude that the main pathophysiological event in the development of streptokinase-induced hypotension lies in the significant reduction in total peripheral resistance as first suggested by Neuhof et al. [10]. Despite the small sample size of 26 patients, the experimental study conducted was supplemented by reliable invasive monitoring devices with high accuracy, such as the Statham transducers to measure the blood pressure in the femoral artery and in the right atrium that represent real-time changes caused by streptokinase in the study subjects. The reduction in total peripheral resistance in patients with streptokinase-induced hypotension is further supported by other reports that consistently found raised complement levels following streptokinase therapy in patients who developed hypotension [4, 11]. Complement activation is known to contribute to widespread vasodilatation in vivo which contributes to a reduction in total peripheral resistance. Despite studies demonstrating clear evidence of the significant rise in anaphylatoxin levels following streptokinase therapy that coincides with the drop in blood pressure [4, 16], more recent papers suggest that there was no direct linear relationship between the degree of complement activation and the magnitude of drop in blood pressure in patients with streptokinase-induced hypotension [11, 17]. This suggests that complement activation unquestionably contributed to the hypotensive episode, but possibly not the primary key player in the underlying mechanism. However, it is worth noting that the study conducted by Pachaii et al. has a small sample size of 13 subjects and that the blood sampling was not concurrently taken during the hypotensive episode [11].

Apart from complement activation, there were a number of mechanisms that have been hypothesized to mediate widespread vasodilatation following streptokinase therapy: (i) direct effect of streptokinase, (ii) activation of the prostaglandin-kallikrein system through factor XII that is activated by plasmin, (iii) FDP-stimulated endothelial prostacyclin secretion, and as part of (iv), of an immunologic reaction to the antigenic streptokinase.

The direct effect of streptokinase in causing vasodilation and the activation of the prostaglandin-kallikrein system has yet to be proven clinically. On the other hand, fibrin-degradation product (FDP) is hypothesized to play a role in platelet activation. Platelet activation is known to trigger the release of serotonin, which in turn stimulates the release of endothelial nitric oxide, a potent vasodilator. However, Taylor et al. in a randomised clinical trial involving 71 patients with acute myocardial infarction found that the use of Lexipafant, a potent platelet-activating factor antagonist did not evade the hypotensive effect of streptokinase therapy [9]. The use of oral medication as the investigational product in this study has an arguable drug pharmacokinetic profile due to the wide age gaps among patients and the potential confounding effect of different gender bias. However, the study did allow for the conclusion of PAF as an insignificant factor in the development of streptokinase-induced hypotension.

Additionally, fibrin degradation product (FDP) has also been suggested to activate the arachidonic acid pathway, releasing prostacyclin, a potent vasodilator. However, an extensive study by Gemmill et al. disproved the role of FDP in the development of hypotension in patients receiving streptokinase therapy as they found no relationship between FDP level and the development of hypotension [7]. However, the study uses pre-medication that includes $100 \mathrm{mg}$ hydrocortisone that may potentially inhibit the arachidonic acid pathway, preventing the release of prostacyclin, further confounding the study results. With regards to the potential inhibition of phospholipase $A_{2}$ enzyme with the use of the corticosteroid, hence potentially preventing hypotension, Pernat et al. did conclude that the maximal drop in the systolic blood pressure was effectively diminished 
with prior use of hydrocortisone with streptokinase therapy [8]. However, the mean difference of $6 \mathrm{~mm} \mathrm{Hg}$ between the treatment group and the control group was too small to be clinically significant despite the statistical significance. Furthermore, evidence suggested that the mechanisms underlying hypotension during streptokinase therapy were most likely multifactorial, hence the use of corticosteroid alone may not entirely prevent the hypotensive episode during streptokinase therapy, but may potentially prevent the maximal drop in blood pressure.

Other studies have also suggested the possible role of antigenicity in the pathophysiology of streptokinase-induced hypotension $[6,18]$. Since streptokinase is harvested from the purified protein of the Group C Streptococcus sp., it carries antigenic property that would trigger the immunological reaction in the recipient. The anti-streptokinase antibodies in the recipient that may have been in the circulation before the administration of streptokinase therapy may interact to stimulate the release of histamine through a cascade of the immunological reaction with the resultant vasodilatory effect. However, Gemmill et al. again disproved this hypothesis as they found no relationship between the hypotensive episode with either baseline anti-streptokinase IgG or streptokinase resistance titre (SKRT) [7].

Apart from systemic vasodilatation, studies have suggested that the fibrinolytic effect of streptokinase may also contribute to the drop in total peripheral resistance by altering the blood viscosity. D-dimer is the breakdown product of cross-linked fibrin, which is a measure of clot lysis, whereas the B- $\beta$ 15-42 peptide is the peptide degradation of fibrin, which is a measure of plasmin activity. Hence, with streptokinase, we would expect that the activation of plasminogen to plasmin will result in increased D-dimer from the breakdown of fibrin and extensive fibrinogenolysis. Since fibrinogen is the main protein linked to blood viscosity, its degradation would have caused the drop in blood viscosity, hence total peripheral resistance, causing a drop in mean arterial pressure. However, Gemmill et al. found no relationship between D-dimer, fibrinogen level and B- $\beta$ 15-42 peptide with the hypotensive episode observed during streptokinase therapy [7].

The mechanism of streptokinase-induced hypotension has not been extensively investigated through clinical trials most likely due to ethical concerns and the limited availability of reliable monitoring and laboratory tests. Therefore, our findings provide grounds for future endeavours to look at possible pharmacological modulation to prevent the hypotensive side effect seen with streptokinase therapy in patients with acute myocardial infarction. Our study is somehow limited in terms of the scoping narrative of the articles as each article carries a different methodological approach, thereby no available articles could be summarised to allow meta-analysis for a solid conclusion. Furthermore, our article search may be limited in view of the initial search strategy having restricted the key terms only to the title/abstract. However, we deemed this search strategy as necessary to narrow down the articles to suit our study focus and prevent streams of irrelevant articles.

Evidently, the limited number of clinical trials available as published literature with regards to streptokinase therapy found in this study is potentially due to the scientific rigor involving human studies that are bound to strict ethical regulations as compared to laboratory-based experiments. Therefore, the mechanism underpinning streptokinase-induced hypotension in patients with acute myocardial infarction was limited to hypothetical evaluation.

In conclusion, our findings suggest that the underlying mechanism of streptokinase-induced hypotension in patients with acute myocardial infarction lies primarily in the reduction in total peripheral resistance, caused by the interplay of both a reduction in whole blood viscosity and the widespread vasodilatation.

\section{Acknowledgments}

The authors would like to thank the Director General of Health Malaysia for his permission to publish this paper.

\section{Conflict of interest}

The authors declare no conflict of interest.

\section{References}

1. Edwards Z, Nagalli S. Streptokinase [Internet]. StatPearls. StatPearls Publishing; 2020. Available from: http://www.ncbi.nlm.nih.gov/pubmed/31985996

2. Aslanabadi N, Safaie N, Talebi F, Dousti S, Entezari-Maleki T. The streptokinase therapy complications and its associated risk factors in patients with acute st elevation myocardial infarction. Iran J Pharm Res 2018; 17 (Special Issue): 53-63.

3. Lateef $F$, Anantharaman V. Hypotension in acute myocardial infarction patients given streptokinase. Singapore Med J 2000; 41: 172-6.

4. Frangi D, Gardinali M, Conciato L, Cafaro C, Pozzoni L, Agostoni A. Abrupt complement activation and transient neutropenia in patients with acute myocardial infarction treated with streptokinase. Circulation 1994; 89: 76-80.

5. Koren G, Weiss AT, Ben-David Y, Hasin Y, Luria MH, Gotsman MS. Bradycardia and hypotension following reperfusion with streptokinase (Bezold-Jarisch reflex): a sign of coronary thrombolysis and myocardial salvage. Am Heart J 1986; 112: 468-71. 
6. Lew AS, Laramee P, Cercek B, Shah PK, Ganz W. The hypotensive effect of intravenous streptokinase in patients with acute myocardial infarction. Circulation 1985; 72: 1321-6.

7. Gemmill JD, Hogg KJ, Douglas JT, et al. The incidence and mechanism of hypotension following thrombolytic therapy for acute myocardixal infarction with streptokinase-containing agents - lack of relationship to pretreatment streptokinase resistance. Eur Heart J 1993; 14: 819-25.

8. Pernat A, Pohar B, Horvat M. Effect of hydrocortisone on streptokinase-induced hypotension in patients with acute myocardial infarction. Fibrinolysis Proteolysis 1997; 11: 103-7.

9. Taylor R, Fatovich D, Hitchcock T, Morrison C, Curtis L. Platelet-activating factor antagonism and streptokinase-induced hypotension in clinical acute myocardial infarction. Clin Sci 2001; 100: 601-7.

10. Neuhof H, Hey D, Glaser E, Wolf H, Lasch HG. Hemodynamic reactions induced by streptokinase therapy in patients with acute myocardial infarction. Eur J Intensive Care Med 1975; 1: 27-30.

11. Pachaii A, Erlendsson AK, Brandslund I. Streptokinase, complement activation and hypotension. APMIS 1997; 105: 650-4.

12. Bendary A, Tawfik W, Mahrous M, Salem M. Fibrinolytic therapy in patients with ST-segment elevation myocardial infarction: accelerated versus standard Streptokinase infusion regimen. J Cardiovasc Thorac Res 2017; 9: 209-14.

13. Chau HW, Choi KK. Efficacy and safety of tenectaplase versus streptokinase in treating st-elevation myocardial infarction patients in Hong Kong: a four-year retrospective review in Queen Elizabeth Hospital. Hong Kong J Emerg Med 2013; 20: 359-63.

14. Arstall MA, Stewart S, Haste MA, Horowitz JD. Streptokinase-induced transient aggravation of myocardial injury. Int J Cardiol 1995; 50: 107-16.

15. Wong CK, White HD, Wilcox RG, et al. New atrial fibrillation after acute myocardial infarction independently predicts death: The GUSTO-III experience. Am Heart 2000; 140: 878-85.

16. Hoffmeister HM, Ruf M, Wendel HP, et al. Activation of the contact phase of the coagulation, of the kinin system and of the complement cascade by streptokinase in acute myocardial infarction. Fibrinolysis Proteolysis 1997; 11 (Suppl. 2): 149-52.

17. Skopál J, Vastag M, Varga L, et al. Streptokinase does not activate the complement system. Blood Coagul Fibrinolysis 2000; 11: 617-22.

18. Dalal J, Sahoo PK, Singh RK, et al. Role of thrombolysis in reperfusion therapy for management of AMI: Indian scenario. Indian Heart J 2013; 65: 566-85. 